\title{
Effect of latanoprost $0.005 \%$ and brimonidine tartrate $0.2 \%$ on pulsatile ocular blood flow in normal tension glaucoma
}

\author{
C Jui-ling Liu, Y-C Ko, C-Y Cheng, J C Chou, W-M Hsu, J-H Liu
}

Br J Ophthalmol 2002;86:1236-1239

\begin{abstract}
Aim: To determine the effect of brimonidine tartrate $0.2 \%$ and latanoprost $0.005 \%$ on pulsatile ocular blood flow (POBF) in patients with normal tension glaucoma (NTG). Method: NTG patients with progressive optic neuropathy, new disc haemorrhage, or field defects that threatened fixation were enrolled into a randomised, investigator masked, crossover study. Group I patients received 4 weeks each of latanoprost, lubricant, and brimonidine, while group II patients received 4 weeks each of brimonidine, lubricant, and latanoprost. Diurnal POBF was measured at baseline and after each 4 week treatment.

Results: 25 patients completed the study and had reliable POBF measurement at each visit. There was no significant diurnal change in baseline POBF $(p=0.768)$. Latanoprost increased POBF by 213 (SD 257) $\mu \mathrm{l} / \mathrm{min}$ (22.8\%, p $<0.001)$ while brimonidine increased it by 97 (183) $\mathrm{\mu l} / \mathrm{min}(10.4 \%, \mathrm{p}=0.014)$. POBF increased at $8 \mathrm{am}(\mathrm{p}=$ $0.004)$, 12 noon $(p=0.002)$, and 4 pm $(p<0.001)$ with latanoprost, while it increased only at $8 \mathrm{am}(p=0.016)$ with brimonidine. After adjusting for the factor of IOP, neither latanoprost nor brimonidine increased POBF significantly.

Conclusions: Latanoprost increases the mean POBF that is related to its IOP lowering effect. The increase in POBF noted after brimonidine is within the range of long term variation and may not be attributable to the drug effect.
\end{abstract}

A ccumulating evidence indicates that intraocular pressure (IOP), even within the normal range, is a major risk factor for normal tension glaucoma (NTG). ${ }^{1-3}$ The risk of visual field progression in NTG is reduced by a substantial decrease of IOP. ${ }^{4}$ On the other hand, attention has been paid to the part vascular factors (that is, decreased blood perfusion or autoregulation dysfunction) play in the pathogenesis of glaucomatous optic neuropathy. ${ }^{6-10}$ Glaucoma medication that lowers IOP but simultaneously reduces ocular blood perfusion may unfavourably influence the disease outcome. ${ }^{11}$ In view of these, the importance of assessing the impact on ocular haemodynamics of a glaucoma medication becomes evident.

The pulsatile ocular blood flow (POBF) tonometer measures the pulsatile component of blood flow that fills the ocular vasculature during cardiac systole. ${ }^{12}{ }^{13}$ POBF accounts for $75 \%$ to $85 \%$ of total ocular blood flow and maintains better capillary perfusion and tissue oxygen metabolism than does nonpulsatile flow. ${ }^{14}$ Choroidal circulation is the major contributor to POBF. Since the main blood supply to the optic disc and the choroid are both from the posterior ciliary arteries, changes in choroidal circulation may be associated with changes in optic disc circulation. This makes measurement of POBF relevant in evaluating ocular haemodynamic effects of glaucoma medications.
Recent studies showed that POBF is enhanced after latanoprost treatment, ${ }^{15-17}$ but it is not enhanced after timolol treatment with comparable IOP reduction. ${ }^{15}$ This indicates that agents may have variable impact on ocular haemodynamics even with the same IOP lowering effect. The influence on POBF of brimonidine is still unknown. The aim of this study was to investigate and compare the effect on POBF after treatment with latanoprost $0.005 \%$ (Xalatan; Pharmacia and Upjohn, Uppsala, Sweden) or brimonidine tartrate $0.2 \%$ (Alphagan; Allergan Inc, Irvine, CA, USA) on NTG patients.

\section{PATIENTS AND METHODS}

NTG patients with progressive optic disc cupping/visual field defects not associated with other disease, new disc haemorrhage, or glaucomatous field defects that threatened fixation were recruited between November 2000 and September 2001. Diagnosis of NTG is based on reproducible field defects of a retinal nerve fibre type (Humphrey visual field analyser, program 24-2), corresponding disc findings, open angles, and normal IOP. IOP was $<22 \mathrm{~mm} \mathrm{Hg}$ on multiple measurements including one diurnal phasing (every 2 hours from 8 am to 8 $\mathrm{pm}$ ). Exclusion criteria were corneal abnormality, a history of corneal surgery, other diseases that might cause field defects, ocular trauma, ocular laser/surgery within 12 months, advanced glaucoma with impaired visual acuity, or severe systemic disease.

Patients taking glaucoma medication before the study underwent a washout period of 4 weeks for topical $\beta$ blockers and latanoprost, 2 weeks for topical adrenergic agonists, and l week for cholinergic agents and carbonic anhydrase inhibitors. ${ }^{18} 19$ Topical lubricant (Artelac; Dr Mann Pharma, Berlin, Germany) was given during the washout period. Patients were then randomly allocated to two groups. Group 1 received latanoprost once daily at $9 \mathrm{pm}$ for 4 weeks, lubricant for 4 weeks, and then brimonidine twice daily at 9 am and 9 pm for 4 weeks. Group 2 followed the same schedule but with the two active regimens in reverse order.

Patients were examined on day 1 , week 4 , week 8 , and week 12. One masked ophthalmologist measured POBF at 8 am, 12 noon, and $4 \mathrm{pm}$ at each visit. On each occasion, POBF was measured three times with one OBF tonometer (Paradigm Medical Industries, Inc, UT, USA). Institutional review board approval was obtained, and verbal and written consent was obtained from all subjects.

The eye with more advanced glaucoma was selected for analysis if both eyes of one patient were eligible. Data were analysed using the statistical software STATA (Stata Corporation, College station, TX, USA). The normality of data distribution was checked with the Shapiro-Wilk test. Comparisons between baseline and after treatment were performed with paired Student's $t$ test. Multivariate linear regression with generalised estimation equation was used to adjust the effects of IOP on POBF. ${ }^{2021}$ Treatment effect 
Table 1 Intraocular pressure $(\mathrm{mm} \mathrm{Hg})$, mean blood pressure $(\mathrm{mm} \mathrm{Hg})$, and pulse rate (beats/min) (mean (SD))

\begin{tabular}{|c|c|c|c|c|}
\hline Measurement & Baseline & After treatment & Change from baseline (\%) & p Value \\
\hline \multicolumn{5}{|c|}{ Intraocular pressure } \\
\hline Latanoprost & $15.5(2.0)$ & $11.8(1.7)$ & $-3.7(1.6)(-23.9 \%)$ & $<0.001$ \\
\hline Brimonidine & $15.5(2.0)$ & $12.9(1.8)$ & $-2.5(1.2)(-16.1 \%)$ & $<0.001$ \\
\hline \multicolumn{4}{|c|}{ ANOVA adjusted difference between the two regimens } & 0.007 \\
\hline \multicolumn{5}{|c|}{ Mean blood pressure } \\
\hline Latanoprost & $91.6(5.9)$ & $91.2(8.3)$ & $-0.4(5.3)(-0.4 \%)$ & 0.703 \\
\hline Brimonidine & $91.6(5.9)$ & $88.2(8.2)$ & $-3.4(7.2)(-3.7 \%)$ & 0.026 \\
\hline \multicolumn{4}{|c|}{ ANOVA adjusted difference between the two regimens } & 0.098 \\
\hline \multicolumn{5}{|l|}{ Pulse rate } \\
\hline Latanoprost & $67.9(9.4)$ & $67.6(10.0)$ & $-0.3(4.5)(-0.4 \%)$ & 0.739 \\
\hline Brimonidine & $67.9(9.4)$ & $65.8(10.4)$ & $-2.1(4.2)(-3.1 \%)$ & 0.021 \\
\hline \multicolumn{4}{|c|}{ ANOVA adjusted difference between the two regimens } & 0.164 \\
\hline
\end{tabular}

Table 2 Pulsatile ocular blood flow $(\mu \mathrm{m} / \mathrm{min})$ at different time point of the day (mean (SD))

\begin{tabular}{|c|c|c|c|}
\hline Measurement & Baseline & After treatment & p Value \\
\hline \multicolumn{4}{|l|}{$8 \mathrm{am}$} \\
\hline Latanoprost & $912.3(251.7)$ & 1115.5 (424.9) & 0.004 \\
\hline Brimonidine & $912.3(251.7)$ & 1027.1 (338.8) & 0.016 \\
\hline \multicolumn{3}{|c|}{ ANOVA adjusted difference between the two regimens } & 0.189 \\
\hline \multicolumn{4}{|l|}{12 noon } \\
\hline Latanoprost & $928.1(270.9)$ & $1165.7(480.1)$ & 0.002 \\
\hline Brimonidine & $928.1(270.9)$ & $1024.4(372.9)$ & 0.064 \\
\hline \multicolumn{3}{|c|}{ ANOVA adjusted difference between the two regimens } & 0.083 \\
\hline \multicolumn{4}{|l|}{$4 \mathrm{pm}$} \\
\hline Latanoprost & $967.5(302.4)$ & $1166.6(379.3)$ & $<0.001$ \\
\hline Brimonidine & $967.5(302.4)$ & $1047.3(376.3)$ & 0.051 \\
\hline \multicolumn{3}{|c|}{ ANOVA adjusted difference between the two regimens } & 0.032 \\
\hline
\end{tabular}

comparisons between two regimens were assessed with a crossover designed analysis of variance (ANOVA). A p value of less than 0.05 was considered as statistically significant.

\section{RESULTS}

Thirty two Asian patients were enrolled; one withdrew after developing a facial rash at the first treatment period. Another six patients were excluded because a digital readout of the POBF results could not be obtained consistently on each occasion at each visit. Data of 25 patients ( 18 men and seven women) with a mean age of 64.4 (SD 12.5) years (range 31-79 years) were analysed. Eighteen patients had no previous glaucoma treatment, and seven patients had been treated with one modality (betaxolol-s in two patients, dorzolamide in one patient, latanoprost in three patients, and laser trabeculoplasty in one patient). Except one eye that received phacoemulsification 10 years before, none had a history of incisional surgery. The mean spherical equivalent was -1.0 (2.5) dioptre (range -6.5 to +3 dioptres). The average visual field mean deviation was -9.66 (7.0) $\mathrm{dB}$ (range -3.08 to $-24.35 \mathrm{~dB}$ ), and the mean pattern standard deviation was 8.73 (4.17) dB (range 2.92 to $16.07 \mathrm{~dB}$ ). No significant side effects were encountered. Ten patients were taking systemic cardiovascular medication ( $\alpha$ blockers in two patients, $\beta$ blockers in three patients, calcium channel blockers in three patients, and angiotensin converting enzyme inhibitors in three patients), and all kept using the same medication during the study.

The data of IOP, mean blood pressure, and pulse rate are shown in Table 1. Brimonidine was associated with a reduction in average mean blood pressure and pulse rate $(\mathrm{p}=$

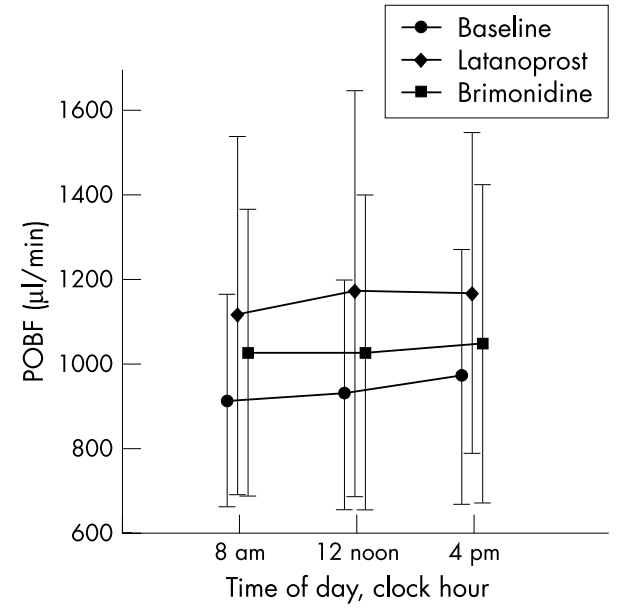

Figure 1 The pulsatile ocular blood flow (POBF) (mean (SD)) at different times of day at baseline and after treatment.

$0.026, p=0.021$, respectively). There was no significant difference $(p=0.768)$ in baseline POBF measured at $8 \mathrm{am}, 12$ noon, and $4 \mathrm{pm}$. POBF increased at all time points with latanoprost, while it increased only at 8 am with brimonidine treatment (Table 2 and Fig 1).

Average POBF increased by $213(257) \mu \mathrm{l} / \mathrm{min}(22.8 \%)$ from a baseline of $936(265) \mu \mathrm{l} / \mathrm{min}(\mathrm{p}<0.001)$ after latanoprost and increased by $97(183) \mu \mathrm{l} / \mathrm{min}(10.4 \%)(\mathrm{p}=0.014)$ after brimonidine treatment. The treatment effect is different in favour of latanoprost $(p=0.04)$. After adjusting for the factor of IOP, the increase of mean POBF associated with both regimens no longer reached statistical significance $(p=0.424$ and $\mathrm{p}=0.345$, respectively).

To avoid the possible effects of systemic cardiovascular medication on POBF, data were further analysed after excluding patients with such medications. The results remained similar. Changes in POBF with latanoprost and brimonidine were not correlated with the visual field mean deviation $(\mathrm{p}=$ $0.987, \mathrm{p}=0.087$, respectively) or pattern standard deviation ( $\mathrm{p}$ $=0.500, \mathrm{p}=0.734$, respectively). No significant period effects or carryover effects were observed.

\section{DISCUSSION}

The measurement of POBF is based on the pressure waveform from continuous IOP recording and a pressure/volume relation as described by Langham and colleagues. ${ }^{12}{ }^{13}$ The validity of the theoretical basis for POBF determination has been shown to be acceptable and its application may result in 
reasonable approximations. ${ }^{22}$ Measurements with the OBF tonometer are reproducible with a coefficient of reliability around $0.92 .^{2324}$

The prostaglandin $\mathrm{F}_{2 \alpha}$ can cause vasoconstriction in isolated bovine retinal arterioles. ${ }^{25}$ Latanoprost, being a prostaglandin $\mathrm{F}_{2 \alpha}$ analogue, might exert vasomotor effects on vessels that supply the optic disc. Similarly, as an $\alpha_{2}$ adrenergic agonist, brimonidine may affect ocular circulation, particularly at the level of terminal arteriole. ${ }^{26}$ We found that the increase in POBF after latanoprost and brimonidine is related to their IOP lowering effect. After adjusting for the factor of IOP, the changes in POBF no longer reached statistical significance. This suggests that either they do not exert a direct vasomotor effect to influence POBF, or the effects are too trivial to be revealed by OBF tonometer. The ocular haemodynamic effects of these agents have been investigated with a variety of methods, such as radioactive/colour microspheres, colour Doppler ultrasonography, and scanning laser Doppler flowmetry. ${ }^{27-31}$ No significant alteration of blood flow or blood velocity was identified in the retina, optic disc, and retrobulbar vessels. None of the studies, including ours, demonstrated that latanoprost or brimonidine impaired ocular circulation in primates.

Our findings with latanoprost agree with previous studies that show an increased POBF after treatment. ${ }^{15-17}$ McKibbin and Menage reported an average POBF increase of $21 \%$ in NTG eyes receiving latanoprost. ${ }^{17}$ Our results are very similar to theirs despite the fact that our patients had a lower baseline IOP. It is noteworthy that non-pulsatile blood flow is not estimated by this technique, and the ratio of pulsatile to non-pulsatile flow may change with alteration in the heart rate, IOP, and systemic blood pressure. Whether an increase in POBF is beneficial to the optic disc remains debatable.

Fontana and colleagues found a lower POBF in eyes of NTG patients with visual field loss than their fellow eyes with normal field, indicating that POBF may provide an indirect estimation of optic disc blood flow. ${ }^{32}$ Furthermore, in a study to evaluate the effect on POBF of trabeculectomy in patients with high tension open angle glaucoma, James found a mean increase of $29 \%$ in POBF after surgery. This suggests that an increased POBF may be associated with favourable prognosis of glaucoma. ${ }^{33}$

To the best of our knowledge, the effect of brimonidine on POBF has not yet been reported. Although a significant increase was identified in our study, the $10.4 \%$ increase after brimonidine treatment may not be attributable to drug effects. One study investigated the variation in repeat measurement of POBF in normal subjects 3 months apart and found a variation up to $12.4 \%$ (Crowhurst $\mathrm{C}$ and Massey $\mathrm{AD}$, data of OBF Labs Ltd), even in experienced hands. The change in POBF should exceed the variability resulting from measurement and physiological variation to be attributable to drug effects.

We noted a statistically significant but clinically insignificant reduction in mean blood pressure (3.4 (7.2) $\mathrm{mm} \mathrm{Hg}$ ) and pulse rate $(2.1(4.2)$ beats/minute) after brimonidine treatment, findings in accordance with several reports. ${ }^{34-36}$ Treatment with $0.2 \%$ brimonidine may reduce the systolic blood pressure up to $7.1 \mathrm{~mm} \mathrm{Hg}$ and decrease the pulse rate up to 3 beats/minute. ${ }^{34-36}$ In a study on ocular normotensive subjects with implanted cardiac pacemakers, Trew et al found that the mean POBF increased by about $14 \%$ as the heart rate increased from 60-90 beats/minute..$^{37}$ Although the decreased pulse rate after brimonidine treatment may in itself reduce POBF, the effect is likely to be insignificant.

In conclusion, 4 week treatment with latanoprost increases POBF, which is related to its IOP lowering effect. The increase in POBF noted after brimonidine is within the range of long term variation and may not be attributable to the drug effect. The clinical significance of enhanced POBF remains to be clarified.

\section{Authors' affiliations}

C Jui-ling Liu, Y-C Ko, C-Y Cheng, J C Chou, W-M Hsu, J-H Liu, Department of Ophthalmology, Taipei Veterans General Hospital, Taipei, Taiwan

C Jui-ling Liu, J C Chou, W-M Hsu, J-H Liu, National Yang-Ming

University School of Medicine, Taipei, Taiwan

Correspondence to: Catherine J Liu, MD, Department of Ophthalmology, Taipei Veterans General Hospital, No 201, Section 2, Shi-Pai Road, Taipei, Taiwan; jlliu@vghtpe.gov.tw

Financial interest: none

Accepted for publication 26 June 2002

\section{REFERENCES}

1 Cartwright MJ, Anderson DR. Correlation of asymmetric damage with asymmetric intraocular pressure in normal-tension glaucoma (low-tension glaucoma). Arch Ophthalmol 1988;106:898-900.

2 Crichton A, Drance SM, Douglas GR, et al. Unequal intraocular pressure and its relation to asymmetric visual field defects in low-tension

3 glaucoma. Ophthalmology 1989;96:1312-4. neuroretinal rim loss in normal-pressure glaucoma. Am J Ophthalmol 1998; 125:137-44.

4 Hitchings RA, Wu J, Poinoosawmy D, et al. Surgery for normal tension glaucoma. Br J Ophthalmol 1995;79:402-6.

5 Collaborative Normal-tension Glaucoma Study Group. Comparison of glaucomatous progression between untreated patients with normal-tension glaucoma and patients with therapeutically reduced intraocular pressures. Am J Ophthalmol 1998;126:487-97.

6 Phelps CD, Corbett JJ. Migraine and low tension glaucoma. Invest Ophthalmol Vis Sci 1985;26:1 105-8.

7 Drance SM, Douglas GR, Wiisman K, et al. Response of blood flow to warm and cold in normal and low-tension glaucoma patients. Am J Ophthalmol 1988;105:35-9.

8 Kaiser HJ, Flammer J, Graef T, et al. Systemic blood pressure in glaucoma patients. Graefes Arch Clin Exp Ophthalmol 1993;231:677-80.

9 Hayreh SS, Zimmerman MB, Podhajsky P, et al. Nocturnal arterial hypotension and its role in optic nerve head and ocular ischemic disorders. Am J Ophthalmol 1994;117:603-24.

10 Graham SL, Drance SM, Wijsman K, et al. Ambulatory blood pressure monitoring in glaucoma: the nocturnal dip. Ophthalmology 1995; 102:61-9.

11 Hayreh SS, Podhajsky P, Zimmerman MB. Beta-blocker eyedrops and nocturnal arterial hypotension. Am J Ophthalmol 1999;1 28:301-9.

12 Langham ME, To'mey K. A clinical procedure for measuring the ocular pulse-pressure relationship and the ophthalmic arterial pressure. Exp Eye Res 1987;27:17-25.

13 Silver DM, Farrell RA, Langham ME, et al. Estimation of pulsatile ocular blood flow from intraocular pressure. Acta Ophthalmol 1989;67(suppl):25-9.

14 Tatsumi E, Miyazaki K, Toda K, et al. Influence of non-pulsatile systemic circulation on tissue blood flow and oxygen metabolism. ASAIO $\mathrm{J}$ 1996;42:M757-62

15 Sponsel WE, Mensah J, Kiel JW, et al. Effects of latanoprost and timolol-XE on hydrodynamics in the normal eye. Am J Ophthalmol 2000;130:151-9.

16 Geyer O, Man O, Weintraub M, et al. Acute effect of latanoprost on pulsatile ocular blood flow in normal eyes. Am J Ophthalmol 2001;131:198-202.

17 McKibbin M, Menage M. The effect of once-daily latanoprost on intraocular pressure and pulsatile ocular blood flow in normal tension glaucoma. Eye 1999;13:31-4

18 Schlecht LP, Brubaker RF. The effects of withdrawal of timolol in chronically treated glaucoma patients. Ophthalmology 1988;95:1212-6.

19 Stewart WC, Holmes KT, Johnson MA. Washout periods for brimonidine $0.2 \%$ and latanoprost 0.005\%. Am J Ophthalmol 2001;131:798-9.

20 Weisberg S. Applied linear regression. 2nd ed. New York: John Wiley, 1985:33-63.

21 Liang KY, Zeger SL. Longitudinal data analysis using generalized linear models. Biometrics 1986;73:13-22.

22 Krakau CET. Calculation of pulsatile ocular blood flow. Invest Ophthalmol Vis Sci 1992;33:2754-6

23 Butt ZA, O'Brien C. Reproducibility of pulsatile ocular blood flow measurements. J Glaucoma 1995:4:214-8.

24 Yang YC, Hulbert MFG, Batterbury M, et al. Pulsatile ocular blood flow measurements in healthy eyes; reproducibility and reference values. J Glaucoma 1997;6:175-9.

25 Hoste AM, Andries L. Contractile responses of isolated bovine retinal microarteries to acetylcholine. Invest Ophthalmol Vis Sci 1991;32:1996-2005.

26 Faber JE, Meininger GA. Selective interaction of alpha-adrenoceptors with myogenic regulation of microvascular smooth muscle. Am J Physiol 1990;259:H1 126-33.

27 Nicolela MT, Buckley AR, Walman BE, et al. A comparative study of the effects of timolol and latanoprost on blood flow velocity of the retrobulbar vessels. Am J Ophthalmol 1996;122:784-9. 
28 Stjernschantz J, Selén G, Astin $M$, et al. Effect of latanoprost on regional blood flow and capillary permeability in the monkey eye. Arch Ophthalmol 1999;117:1363-7.

29 Seong GJ, Lee HK, Hong YJ. Effects of $0.005 \%$ latanoprost on optic nerve head and peripapillary retinal blood flow. Ophthalmologica 1999:213:355-9.

30 Lachkar Y, Migdal C, Dhanjil S. Effect of brimonidine tartrate on ocular hemodynamic measurements. Arch Ophthalmol 1998;116:1591-4.

31 Carlsson AM, Chauhan BC, Lee AA, et al. The effect of brimonidine tartrate on retinal blood flow in patients with ocular hypertension. Am J Ophthalmol 2000;129:297-301.

32 Fontana L, Poinoosawmy D, Bunce CV, et al. Pulsatile ocular blood flow investigation in asymmetric normal tension glaucoma and normal subjects. Br J Ophthalmol 1998;82:731-6.
33 James CB. Effect of trabeculectomy on pulsatile ocular blood flow. $\mathrm{Br}$ J Ophthalmol 1994;78:818-22

34 Nordlund JR, Pasquale LR, Robin AL, et al. The cardiovascular, pulmonary, and ocular hypotensive effects of $0.2 \%$ brimonidine. Arch Ophthalmol 1995;113:77-83.

35 Serle JB, the Brimonidine Study Group III. A comparison of the safety and efficacy of twice daily brimonidine $0.2 \%$ versus betaxolol $0.25 \%$ in subjects with elevated intraocular pressure. Surv Ophthalmol 1996;41(Suppl 1): S39-47.

36 Derick RJ, Robin AL, Walters TR, et al. Brimonidine tartrate: a one-month dose response study. Ophthalmology 1997;104:131-6.

37 Trew DR, James CB, Thomas SHL, et al. Factors influencing the ocular pulse-the heart rate. Graefes Arch Ophthalmol 1991;229:553-6.

\section{$\mathrm{ECHO}$}

\section{B cells may be key cells in uveitis}

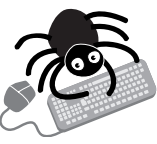

Please visit the British Journal of

Ophthalmology website [www. bjophthalmol. com] for link to this full article. ontrolling the immune response might be a way of controlling uveitis, a small observational study has suggested. A team of specialists from different disciplines has found that patients with uveitis of unknown origin have significantly more CD5 + B cells circulating in their blood than healthy controls (mean (SD) amounts $91(76) \times 10^{6} / 1$ in patients and $58(34) \times 10^{6} / 1$ in controls).

This was also true for patients who had anterior uveitis $\left(94(79) \times 10^{6} / 1\right)$ and severe uveitis $(108(93)$ $\times 10^{6} / 1$ ), though the values did not differ significantly between each other. No appreciable differences were seen between anterior and posterior uveitis, uveitis in one or both eyes, or single or multiple episodes.

Twenty seven patients were tested out of 315 with uveitis not associated with any systemic diseases and compared with 21 healthy age and sex matched controls. Twenty patients had anterior uveitis and seven posterior uveitis.

High numbers of CD5 + B cells are found in some autoimmune diseases, where they seem to have a role in pathogenesis. Uveitis may be of unknown origin or associated with many general diseases and is assumed to have an immunological component. 